\title{
Aspectos clínicos da ruptura do músculo gastrocnêmio em ruminantes: descrição de três casos
}

\author{
[Clinical aspects of rupture of the gastrocnemius muscle in ruminants: description of three cases]
}

\section{"Relato de Caso/Case Report"}

\author{
Luiz Teles Coutinho, Ângela Imperiano da Conceição*, Nivaldo de Azevedo Costa, Nivan \\ Antônio Alves da Silva, Maria Isabel de Souza, Carla Lopes de Mendonça, Rodolfo José \\ Cavalcanti Souto, Jobson Filipe de Paula Cajueiro, José Augusto Bastos Afonso
}

Clínica de Bovinos de Garanhuns, Universidade Federal Rural de Pernambuco, Garanhuns-PE, Brasil.

*Autor para correspondência/Corresponding author: E-mail: angelaimperiano@ hotmail.com

\begin{abstract}
Resumo
Esse trabalho tem por finalidade descrever a ocorrência e os aspectos clínicos da ruptura do músculo Gastrocnêmio em três animais (um bovino, um caprino e uma lhama) de idades diferentes, fêmeas, criados de forma semi-intensiva em propriedades distintas, que foram atendidos na Clínica de Bovinos de Garanhuns, Universidade Federal Rural de Pernambuco, cujos proprietários queixavam-se de problema locomotor. Essa enfermidade é rara ou incomum na clínica de ruminantes e, portanto, ainda pouco relatada. As principais causas relacionadas são os traumas e neuropatias pós-parturiente, sendo o diagnóstico geralmente clínico, baseandose na identificação e no reconhecimento das alterações musculoesqueléticas e da postura, que são características. Os achados clínicos encontrados nos animais atendidos foram incapacidade elou dificuldade de levantar e ficar em estação, alteração na marcha, postura característica e inflamação local. Em dois animais a ruptura do músculo foi total e no outro a lesão foi parcial. Diante do prognóstico desfavorável indicou-se o abate do caprino e, em detrimento da idade da lhama, foi instituído tratamento com utilização de antiinflamatórios não esteroides, porém sem sucesso. No caso do bovino, sugeriu-se a permanência temporária do animal, em espaço restrito, a fim de aproveitar a sua produção láctea. Dada à importância funcional desse músculo, sua ruptura compromete a viabilidade produtiva do animal afetado.
\end{abstract}

Palavras-chave: claudicação; decúbito; doença muscular.

\begin{abstract}
The aim of this study is to describe the occurrence and clinical aspects of gastrocnemius muscle rupture in three animals (a bovine, a goat, and a llama), of different ages, female, raised under a semi-intensive system on different farms. All the animals presented to the Clínica de Bovinos of Garanhuns, Federal Rural University of Pernambuco, with a complaint of locomotor disorder. As this disease is rare or unusual in ruminants, its occurrence is rarely reported. Traumas and postpartum neuropathies are the most commonly reported causes. The diagnosis is often clinical and is based on identification and recognition of musculoskeletal and postural changes, which are characteristics of the disease. The clinical findings were disability and/or difficulty in rising and standing, changes in gait, characteristic posture and local inflammation. In two animals, the rupture of the muscle was complete and in the other, it was partial. In view of the unfavorable prognosis, the slaughter of the goat was suggested and, despite the age of the llama, nonsteroidal anti-inflammatory drugs were administered, but without success. In the case of the bovine, it was suggested the animal be kept temporarily in a restricted space to take advantage of its milk production. Given the functional importance of this muscle, its rupture compromises the productive viability of the affected animal.
\end{abstract}

Keywords: decubitus; lameness; muscle disease. 


\section{Introdução}

O músculo Gastrocnêmio é responsável pela flexão do joelho e extensão do jarrete; funções essas de crucial importância para o "levantar", a sustentação e a locomoção dos animais (Getty, 1981; Divers e Peek, 2018). Sendo assim, lesões acometendo esse músculo podem, a depender da gravidade e extensão, resultar no comprometimento dessas atividades (Dirksen e Doll, 2005).

As principais causas relacionadas com a ruptura do músculo Gastrocnêmio são os traumas, oriundos de acidentes diversos, e as neuropatias pós-parturiente (Dirksen e Doll, 2005; Anderson et al., 2008; Cardona et al., 2017; Mori et al., 2017; Divers e Peek, 2018). Essas causas são potencializadas por fatores predisponentes, como as alterações metabólicas do periparto (hipocalcemia, por exemplo) e as compressões por decúbito prolongado (Dirksen e Doll, 2005; Mori et al., 2017; Divers e Peek, 2018), que, em interação, podem favorecer o surgimento da lesão.

Geralmente o diagnóstico de ruptura do Gastrocnêmio é clínico, e baseia-se na identificação e no reconhecimento das alterações musculoesqueléticas e da postura, que são características (Dirksen e Doll, 2005; Divers e Peek, 2018; Cardona et al., 2017; Mori et al., 2017). Todavia, pode-se também utilizar, como ferramenta auxiliar, a ultrassonografia (Tyler et al., 1998; Kwak et al., 2006). Na maioria dos casos, quando a ruptura é total, não há recuperação e o prognóstico dos animais acometidos é desfavorável (Greenough e Weaver, 1997; Dirksen e Doll, 2005).

Por ser uma enfermidade rara ou de ocorrência incomum e, consequentemente, ainda pouco descrita na clínica de grandes animais, o objetivo desse trabalho é descrever a ocorrência e os aspectos clínicos da ruptura do músculo Gastrocnêmio em três espécies de ruminantes.

\section{Descrição do Caso}

Três ruminantes (um bovino, um caprino e uma lhama) de idades diferentes, fêmeas, criados semi-intensivamente em propriedades distintas, foram atendidos pela Clínica de Bovinos de Garanhuns, Universidade Federal Rural de Pernambuco (CBG-UFRPE), cujos proprietários queixavam-se de problema locomotor. O histórico do bovino foi que, após o parto ocorrido há 2,5 meses, apresentou dificuldade em estender completamente o membro posterior direito (MPD); o caprino, há quatro dias, permanecia muito tempo em decúbito e relutava em levantar. A lhama, nascida há cinco dias de parto distócico, não conseguia ficar em estação.

Os exames clínicos do bovino e do caprino foram realizados na CBG-UFRPE, enquanto que a lhama foi atendida na propriedade rural a qual pertencia (Dirksen et al., 1993). O bovino apresentava marcha insegura, claudicação mista grau III e semiflexão na articulação do boleto do membro pélvico direito. Quando em estação/repouso, o animal posicionava o membro afetado cranialmente em relação ao contralateral, mantendo o jarrete "caído" (mais baixo) e o boleto, permanentemente, semiflexionado (Figura 1). Essas alterações caracterizam o quadro de ruptura parcial do músculo Gastrocnêmio e foram, identicamente, descritas e/ou observadas em animais com essa lesão (Dirksen e Doll, 2005), que são menos frequentes que a ruptura total (Divers e Peek, 2018).

No caprino e na lhama, foi observada a clássica apresentação "perna de coelho" (Figura 2), patognomônica da ruptura total do músculo Gastrocnêmio (Dirksen e Doll, 2005; Divers e Peek, 2018), onde o jarrete, apoiado no solo, forma um ângulo de 90 graus entre a tíbia e o metatarso. Essa postura, que também reflete a gravidade da lesão (Venkatesakumar et al., 2013), pode ser melhor identificada quando o clínico se posiciona atrás do animal e o observa levantar. Entretanto, em se tratando de animais muito pesados e/ou de lesões bilaterais, isso nem sempre será possível (Dirksen e Doll, 2005; Divers e Peek, 2018). Outras alterações observadas foram decúbito prolongado, relutância e dificuldade em levantar, além de sinais de inflamação - tumefação e sensibilidade - na musculatura posterior do membro afetado. Demais variáveis, como coloração das mucosas, temperatura corporal, apetite, dinâmica de rúmen e frequência cardiorrespiratória encontravam-se na faixa de normalidade para as espécies.

\section{Discussão}

Essas alterações sugerem a ocorrência de traumas como a possível causa da lesão muscular no bovino e no caprino; e mesmo sem indícios destes traumas terem ocorrido, eles são intimamente associados aos casos de ruptura da unidade músculo-tendão Gastrocnêmio (Anderson et al., 2008). Reitera-se ainda que, distensões e/ou trações exageradas são também causas potenciais 
desse tipo de injúria muscular (Venkatesakumar et al., 2013; Divers e Peek, 2018) e, provavelmente, foi o que ocorreu com a lhama, a qual nasceu de laboriosa distorcia manipulada por pessoal inabilitado. Além do mais, no caso do bovino desse relato, há de se considerar que a lesão possa ter sido potencializada por algum dos fatores predisponentes associado ao periparto, como a hipocalcemia, por exemplo (Getty, 1981; Dirksen e Doll, 2005; Mori, et al., 2017).

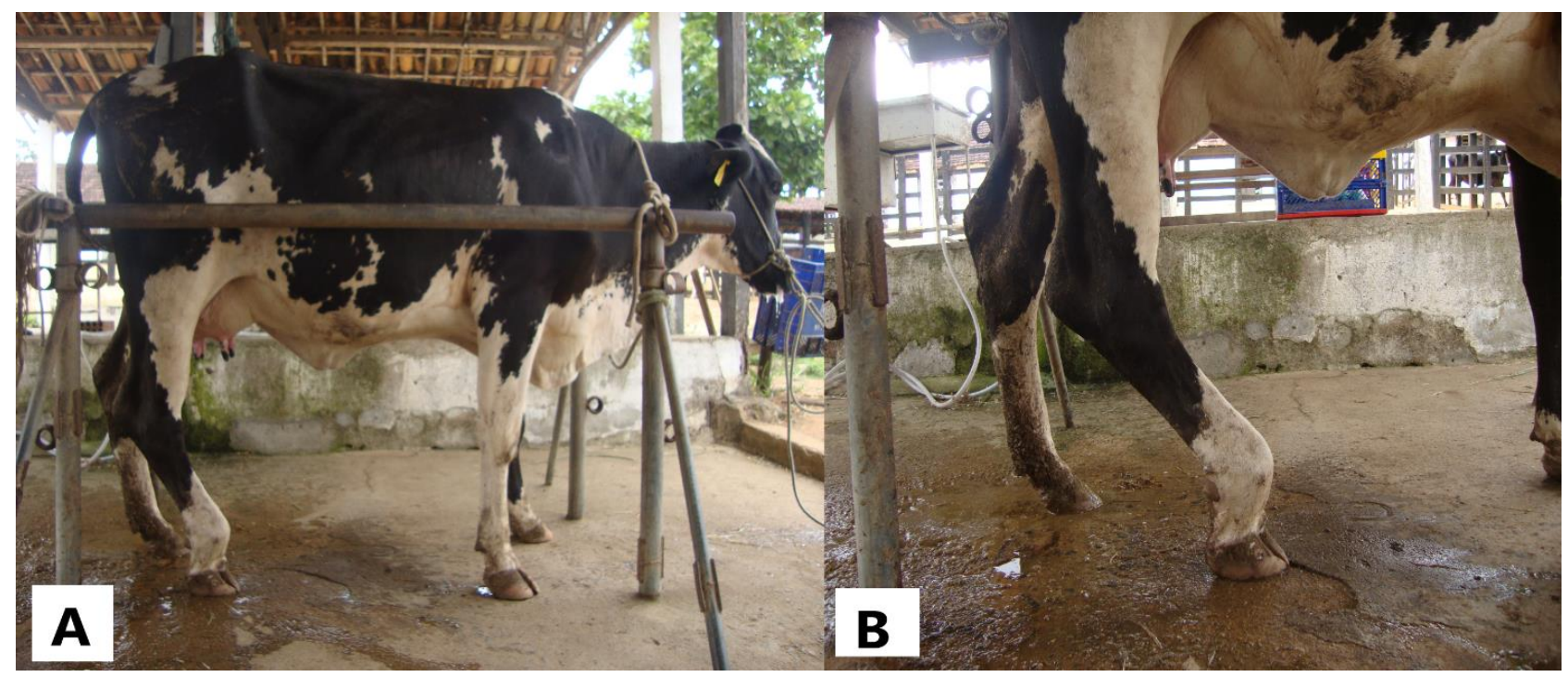

Figura 1. (A) bovino com ruptura parcial do músculo Gastrocnêmio. Disposição do membro pélvico direito mais a frente, com o jarrete "caído" e a articulação do boleto, permanentemente, semiflexionada; (B) imagem aproximada da Figura A, mostrando mais definidamente os detalhes do jarrete e boleto no membro afetado.

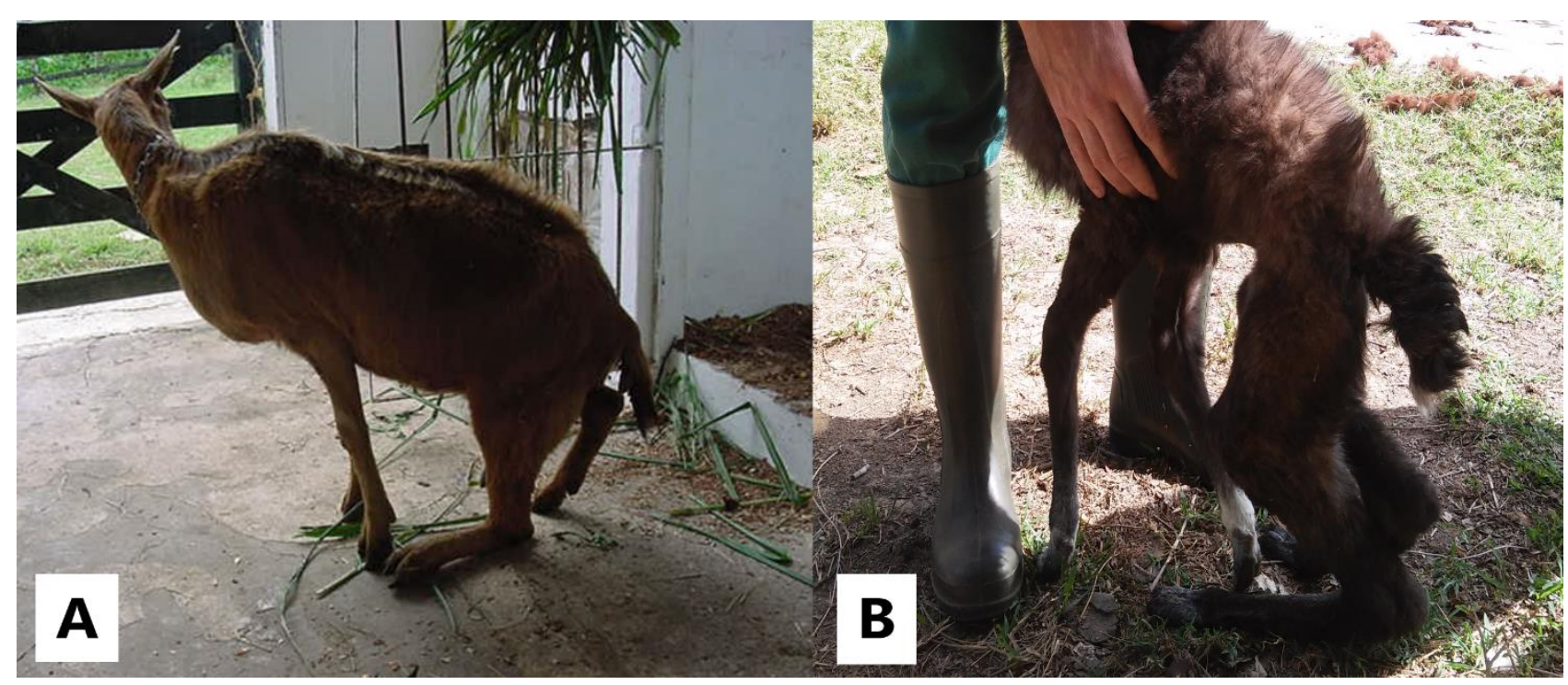

Figura 2. (A) caprino e (B) lhama, respectivamente, com ruptura total do músculo Gastrocnêmio esquerdo. Observa-se a clássica apresentação "perna de coelho", onde o jarrete apoiado no solo permite a formação de um ângulo de 90 graus entre a tíbia e o metatarso.

Em casos de ruptura total, como a que ocorreu no caprino e na lhama, comprometendo a viabilidade produtiva (Greenough e Weaver, 1997; Dirksen e Doll, 2005), justifica-se a conduta de indicar os animais ao abate. Em virtude de a lhama ser recém-nascida, foi realizada tentativa terapêutica com utilização de anti-inflamatórios não esteroides e repouso, porém sem recuperação desejada, divergindo de resultados obtidos em outros trabalhos que relatam sucesso no tratamento de casos de ruptura do músculo Gastrocnêmio (Tyler et al., 1998; Vlamynck et al., 2016). No bovino, como a lesão foi menos severa (rompimento parcial), permitiu a permanência temporária do animal, em espaço restrito, a fim de aproveitar a sua produção láctea. 


\section{Conclusão}

Dada a importância funcional do músculo Gastrocnêmio, é fundamental o diagnóstico de sua ruptura que, a depender do tipo e gravidade da lesão, pode comprometer a viabilidade produtiva do animal, e assim evitar prejuízos econômicos ainda maiores e desnecessários aos produtores.

\section{Conflito de Interesse}

Os autores declaram não existir conflito de interesse.

\section{Referências}

Anderson, D.E.; Desrochers, A.; Jean, G.S. Management of tendon disorders in cattle. Veterinary Clinics: Food Animal Practice, 24(3): 551-566, 2008.

Cardona, J.A.; Suarez, A.C.H.; Isaías, J.A. Rupture of the gastrocnemius muscle in a heifer Gyr in the department of Córdoba, Colombia. Revista Colombiana de Ciencia Animal, 9(1): 94-97, 2017.

Dirksen, G.; Doll, K. Enfermidades del aparato locomotor. In: Medicina interna $y$ cirugía del bovino. $4^{\mathrm{a}}$ ed. Buenos Aires: InterMédica; 2005. p. 683-932.

Dirksen, G.; Gründer, H.D.; Stöber, M. Rosenberger: exame clínico dos bovinos. $3^{\mathrm{a}}$ ed. Rio de Janeiro: Guanabara Koogan, 1993. $419 \mathrm{p}$.

Divers, T.J.; Peek, S.F. Rebhun's: diseases of dairy cattle. $3^{\text {rd }}$ ed. St. Louis: Saunders Elsevier, 2018. 704p.
Getty, R. Anatomia dos animais domésticos. $5^{\mathrm{a}}$ ed. Rio de Janeiro: Interamericana, 1981. $1134 p$.

Greenough, P.R.; Weaver, A.D. Lameness in cattle. $3^{\text {rd }}$ ed. Philadelphia: W.B. Saunders Elsevier, 1997. 336p.

Kwak, H.; Han, Y.; Lee, S.; Kim, K.; Chung, G.H. Diagnosis and follow-up us evaluation of ruptures of the medial head of the gastrocnemius (Tennis Leg). Korean Journal of Radiology, 7(3): 193-198, 2006.

Mori, A.P.; Schwertz, C.I.; Henker, L.C.; Stedille, F.A.; Christ, R.; Lorenzett, M.P.; Broll, F.; Mendes, R.E. Bilateral gastrocnemius muscle rupture in a bovine. Acta Scientiae Veterinariae, 45(Suppl. 1): 189-193, 2017.

Tyler, J.W.; Middleton, J.R.; Barbee, D.D.; Parish, S.M. Conservative management of a ruptured gastrocnemius muscle in a male llama. Canadian Veterinary Journal, 39(11): 712713, 1998.

Venkatesakumar, E.; Sivaraman, S.; Ponnuswamy, K.K.; Pandian, S.J.; Vijayakumar, G.; Subramanian, M. Rupture of the gastrocnemius muscle in a cow. Indian Veterinary Journal, 90(12): 65-66, 2013.

Vlamynck, C.; Pille, F.; Vlaminck, L. Long term outcome of conservative management or surgical treatment of bovine spastic paresis: 79 cases. Veterinary Surgery, 45(2): 187-193, 2016. 